\title{
Lesson of the month: Late, disseminated herpes zoster reactivation in a stem cell transplant recipient: implications for post-transplant prophylaxis and immune monitoring
}

\author{
Authors: Amy E Edwards, ${ }^{A}$ Cristina Suarez ${ }^{B}$ and Jonathan Lambourne ${ }^{C}$
}

\begin{abstract}
Varicella zoster reactivation is a known risk following stemcell transplantation, but has become more infrequent since universal antiviral prophylaxis. We report an unusual case of late, disseminated reactivation in a 27 -year-old man with positive pre-transplant serology, and discuss implications for post-transplant prophylaxis and immune monitoring.
\end{abstract}

KEYWORDS: herpes zoster, varicella zoster, stem cell transplant, viral reactivation, cellular immunity

DOI: 10.7861/clinmed.2021-0064

\section{Case presentation}

A 27-year-old man presented with diffuse abdominal pain, nausea and constipation. Abdominal imaging revealed oedematous pancreatitis and non-obstructive cholecystitis. Eight months earlier, he had undergone myeloablative, haplo-identical, allogeneic stem cell transplantation (SCT) for treatment of acute lymphoblastic leukaemia (ALL). Post-transplant immunosuppression had been stopped 4 months prior to admission and his regular medication comprised ranitidine (150 mg once daily (od)), and prophylactic aciclovir (400 mg twice daily (bd)), co-trimoxazole (960 mg three times a week) and phenoxymethylpenicillin ( $250 \mathrm{mg} \mathrm{bd}$ ).

Intravenous (IV) fluids and antibiotics (co-amoxiclav and gentamicin) were commenced. Two days into his admission, a painless, non-pruritic, vesicular rash emerged, which spread from his face to involve his trunk, arms, thighs and hard palate (Fig 1). He also developed profuse diarrhoea. On examination, he was febrile $\left(39.6^{\circ} \mathrm{C}\right)$ and appeared unwell. His pulse rate was 102 beats/min and blood pressure was 102/64 mmHg. Heart sounds were normal, and his chest was clear on auscultation. His abdomen was soft with generalised tenderness and active bowel sounds. There were no focal neurological signs and examination of the eyes was unremarkable. There was a diffuse rash affecting his face, trunk and limbs but sparing palmar and plantar surfaces; lesions were dark, haemorrhagic vesicles of $1-4 \mathrm{~mm}$ diameter over

Authors: ${ }^{A}$ clinical teaching and research fellow, Barts Health NHS Trust, London, UK; ${ }^{B}$ speciality trainee in infectious diseases and microbiology, Barts Health NHS Trust, London, UK; ' ${ }^{C}$ consultant in infectious diseases and microbiology, Barts Health NHS Trust, London, UK an erythematous base (Figs 1a and b). Pigmented lesions were also noted over the hard palate, with some ulceration (Fig 1c).

Admission blood tests are shown in supplementary material S1. These were remarkable for hyponatraemia (sodium of $125 \mathrm{mmol} / \mathrm{L}$ ), hypocalcaemia (adjusted calcium of $2.11 \mathrm{mmol} / \mathrm{L}$ ) and marked transaminitis. C-reactive protein (CRP) and lipase were mildly elevated at $42 \mathrm{mg} / \mathrm{L}(<4 \mathrm{mg} / \mathrm{L})$ and $98 \mathrm{U} / \mathrm{L}(<60 \mathrm{U} / \mathrm{L})$, respectively.

With concerns that his presentation may have been due to graft versus host disease (GvHD), IV methylprednisolone was commenced at $1 \mathrm{mg} / \mathrm{kg}$ od. Co-amoxiclav was changed to piperacillin-tazobactam to cover the possibility of gut translocation in the setting of acute gastrointestinal GvHD and in case the skin lesions were ecthyma gangrenosum. He was also started on IV aciclovir $10 \mathrm{mg} / \mathrm{kg}$ three times daily (tds) in case the skin lesions were due to herpes simplex virus (HSV) or varicella zoster virus (VZV). Given that the patient had previously been a resident in the tropics, he was also given a dose of ivermectin in case this presentation was due to disseminated infection with Strongyloides stercoralis. Swabs of skin and palatal lesions were sent for viral polymerase chain reaction (PCR) and bacterial culture and an extensive infection screen was completed, including enterovirus, adenovirus, herpes PCR and biopsy of one of the skin lesions (supplementary material S2).

Investigations confirmed disseminated VZV infection, with identification of VZV viraemia and positive VZV PCR from skin and palatal lesion swabs. The abdominal symptoms and skin rash improved after several days of aciclovir treatment, and methylprednisolone was weaned. Antimicrobials were deescalated after 7 days and prophylactic co-trimoxazole and phenoxymethylpenicillin recommenced. He was discharged on the 14th day after admission.

The patient had had no unwell contacts and no contact with children before admission. He did not recall a history of chickenpox but had positive VZV serology both pre- and post-transplant, with titres of 3,320 and $182 \mathrm{mIU} / \mathrm{mL}$, respectively, at levels deemed to represent adequate humoral immunity. Further immunological investigation revealed a CD4+ lymphopenia, with an absolute CD4+ count of $56 \times 10^{6} / \mathrm{L}$ (normal range 455-1,320; supplementary material S3).

\section{Discussion}

VZV reactivation is a significant risk following haematopoietic $(H)$ SCT. Rates of $20-45 \%$ during the first year post-transplant were reported in the absence of antiviral prophylaxis, depending upon 

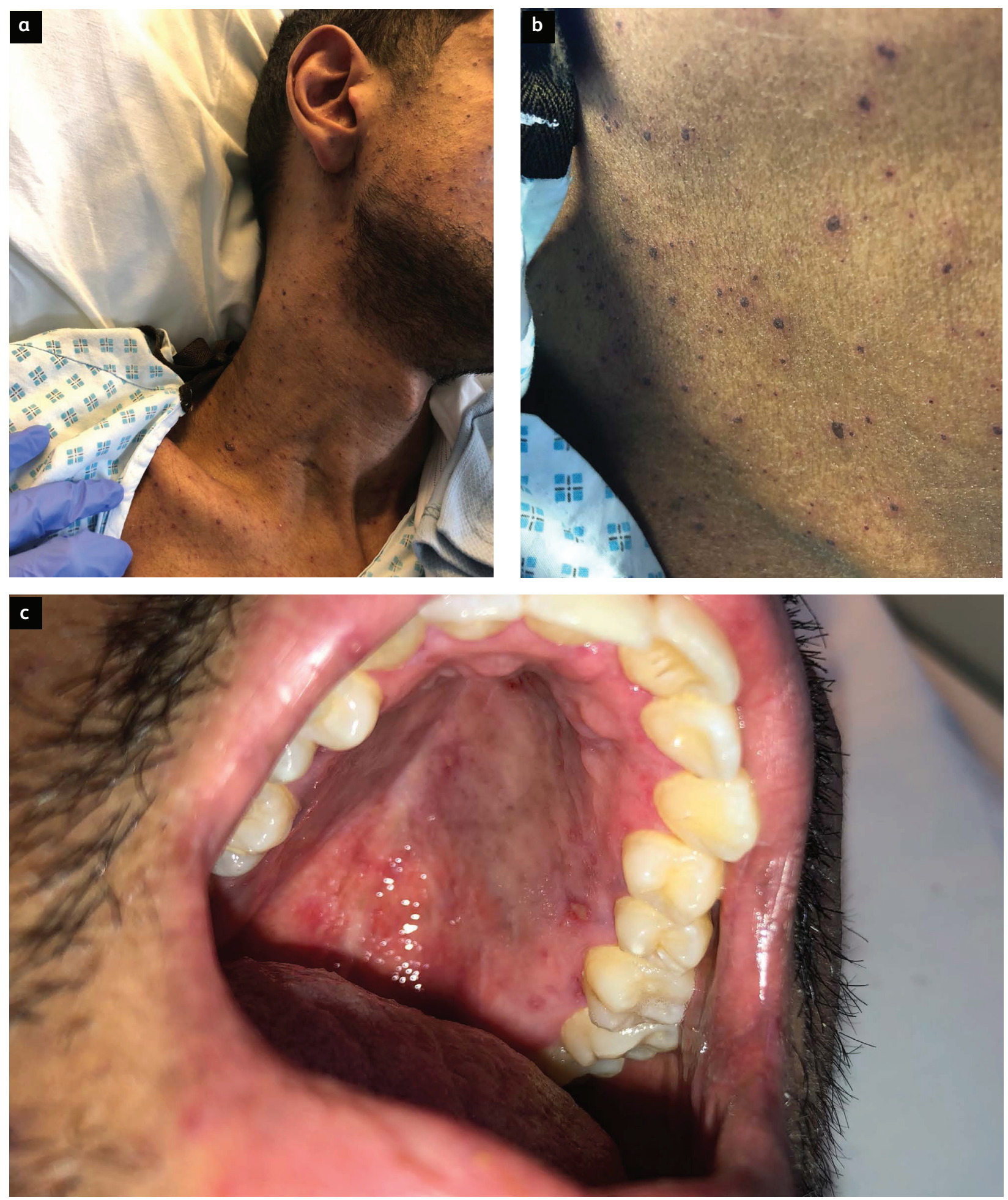

Fig 1. A diffuse painless, non-pruritic, vesicular rash consisting of dark 1-4 mm haemorrhagic vesicles. a) Rash across the face, neck and chest. b) Close-up image of the vesicles. c) Pigmented, ulcerated lesions on the hard palate.

transplant conditioning and the presence or absence of GvHD. ${ }^{2-4}$ The majority of reactivation occurs within 9-12 months, although cases of herpes zoster $(\mathrm{HZ})$ have been reported as late as 3 years post-transplant. ${ }^{3,5}$ Antiviral prophylaxis with aciclovir is now universal post-transplant and has reduced first-year reactivation rates to $2-5 \%$. VZV reactivation typically presents as dermatomal $\mathrm{HZ}$, but less commonly manifests as disseminated infection with widespread cutaneous, mucocutaneous and, at times, visceral involvement. 
Visceral involvement in disseminated VZV has been reported but is a challenge to diagnose. Acute severe abdominal pain may be the presenting feature of visceral infection. ${ }^{6}$ Abdominal symptoms (most commonly epigastric pain, nausea, vomiting, diarrhoea, and elevated hepatic and pancreatic enzymes) can precede skin eruptions by several days and may also occur in the absence of cutaneous disease. ${ }^{7,8}$ Neurological and respiratory manifestations of disseminated zoster are also important to identify, the latter accounting for most fatal cases of disseminated disease. ${ }^{9}$

Clinical diagnosis of disseminated zoster post-transplant can be challenging, as immune suppressed patients are more likely to present with atypical mucocutaneous or visceral disease that may mimic other infections, drug reactions or GvHD. ${ }^{10} \mathrm{As}$ for our patient, laboratory testing is becoming the standard for confirmation in such cases. Although VZV was not definitively confirmed as the cause of abdominal symptoms in the case we present, it is likely that, in the context of severe disseminated viral reactivation and without alternative explanation, these symptoms represented visceral VZV infection.

Primary VZV infection induces both humoral and cellular immune responses. ${ }^{11}$ Cell-mediated immunity is more important than humoral in preventing viral reactivation. ${ }^{12}$ This is reflected in the increase in rate of $\mathrm{HZ}$ with advancing age in the immunocompetent population, as although antibody titres remain relatively stable over time, virus-specific cell-mediated immunity wanes. ${ }^{13}$ Specifically, a VZV-specific T-cell response is required to prevent symptomatic reactivation of endogenous virus, as well as infection after exogenous re-exposure. ${ }^{11,13}$ There is growing evidence for a predominant $\mathrm{CD} 4+\mathrm{T}$-cell response in VZV infection, and a proven role of CD4+ cells in suppressing viral replication. ${ }^{14}$ However, as yet, there is no reliable way to measure VZV-specific cell-mediated immunity and so the presence of humoral immunity in the form of an anti-VZV antibody titre is used as a surrogate. This case highlights that serum VZV immunoglobulin ( $\mathrm{Ig}$ ) $\mathrm{G}$ is not an entirely reliable indicator of protection against reactivation, and that the presence of VZV IgG should not dissuade clinicians from considering the diagnosis of VZV reactivation.

Antiviral prophylaxis with aciclovir is now universal posttransplant and has been demonstrated to significantly reduce VZV reactivation risk. ${ }^{15}$ However, as highlighted in this case, severe infection due to cutaneous and disseminated reactivation may still occur despite prophylaxis. 'The reasons for this are unclear, and may include lack of adherence, impaired absorption and evolution of antiviral resistance. Most cases of VZV reactivation occur following discontinuation of antiviral prophylaxis, with reactivation rates of $31-33 \%$ reported within 3 months of discontinuation. ${ }^{4,16}$ In the absence of a test to measure VZVspecific cell-mediated immunity, adopting an approach based on CD $4+$ cell count recovery may offer a more nuanced and biologically relevant approach than the current time-based strategy for discontinuing aciclovir prophylaxis. T-cell immune reconstitution may be expected around 3-4 months following allogeneic SCT, with CD4+ cells known to reconstitute later than $\mathrm{CD} 8+{ }^{17}$ However, this is highly variable, as demonstrated in our patient in whom reconstitution was incomplete at 8 months post-transplant. This raises the questions of whether lymphocyte subsets should be measured routinely, and if the duration of antiviral prophylaxis should be extended until complete reconstitution, with $\mathrm{CD} 4+$ count consistently greater than $200 \times 10^{6} / \mathrm{L}$ is confirmed. ${ }^{4,18}$

\section{Key points}

> Severe, disseminated VZV reactivation is possible in the context of post-transplant immunocompromise, even with positive serology, ongoing antiviral prophylaxis and following the cessation of immunosuppressive therapy.

$>$ High clinical suspicion is necessary for diagnosis of disseminated infection in such cases, both in the presence and absence of a characteristic skin eruption; with PCR of vesicle fluid and/or blood samples being the key diagnostic test.

> Abdominal symptoms may be the first and, sometimes, only clinical feature of disseminated VZV infection, and visceral VZV reactivation should be on the differential diagnosis list for any patient post-transplant with abdominal pain and/or a transaminitis; with a PCR on ethylenediaminetetraacetic acid (EDTA) blood being the key diagnostic test.

> Lymphocyte subsets should be measured in transplant patients presenting with possible unusual infections, as the degree of immune compromise will influence the range of potential pathogens and should influence the use of antimicrobial prophylaxis.

> There may be an argument for extending aciclovir prophylaxis in patients with positive pre-transplant serology until they have a CD4+ cell count consistently greater than $200 \times 10^{6} / \mathrm{L}$. Tests of pathogen-specific cell-mediated immunity are now available for cytomegalovirus, and a similar assay for VZV would be helpful.

\section{Supplementary material}

Additional supplementary material may be found in the online version of this article at www.rcpjournals.org/clinmedicine: S1 - Haematology and biochemistry results on admission. S1 - Summary of infection investigation results. S1 - Summary of immunological investigations.

\section{Acknowledgements}

The authors would like to thank the patient for allowing us to publish this case report.

\section{References}

1 Baumrin E, Cheng M, Kanjilal S et al. Severe herpes zoster requiring intravenous antiviral treatment in allogeneic hematopoietic cell transplantation recipients on standard acyclovir prophylaxis. Biol Blood Marrow Transplant 2019:25:1642-7.

2 Arvin AM. Varicella-zoster virus infections. In: Thomas ED, Blume KG, Forman SJ. Hematopoietic cell transplantation, 2nd edn. Blackwell Science, 1999: 591-606.

3 Lee CJ, Savani BN, Ljungman P. Varicella zoster virus reactivation in adult survivors of hematopoietic cell transplantation: how do we best protect our patients? Biol Blood Marrow Transplant 2018;24:1783-7.

4 Thomson KJ, Hart DP, Banerjee L et al. The effect of low-dose aciclovir on reactivation of varicella zoster virus after allogeneic haemopoietic stem cell transplantation. Bone Marrow Transplant 2005:35:1065-9.

5 Locksley RM, Flournoy N, Sullivan KM, Meyers JD. Infection with varicella-zoster virus after marrow transplantation. J Inf Dis 1985;152:1172-81.

6 Yagi T, Karasuno T, Hasegawa T et al. Acute abdomen without cutaneous signs of varicella zoster virus infection as a late complication of allogeneic bone marrow transplantation: importance 
of empiric therapy with acyclovir. Bone Marrow Transplant 2000;25:1003-5.

7 David DS, Tegtmeier BR, O'Donnell MR, Paz IB, McCarty TM. Visceral varicella-zoster after bone marrow transplantation: report of a case series and review of the literature. Am J Gastroenterol 1998;93:810-3.

8 Koc Y, Miller KB, Schenkein DP et al. Varicella zoster virus infections following allogeneic bone marrow transplantation: frequency, risk factors, and clinical outcome. Biol Blood Marrow Transplant 2000;6:44-9.

9 Feldman S. Varicella-zoster virus pneumonitis. Chest 1994;106:22S-7S

10 Wang L, Verschuuren EAM, van Leer-Buter CC et al. Herpes zoster and immunogenicity and safety of zoster vaccines in transplant patients: a narrative review of the literature. Front Immunol 2018:9:1632.

11 Laing KJ, Ouwendijk WJD, Koelle DM, Varjans G. Immunobiology of varicella-zoster virus infection. J Infect Dis 2018;218:S68-74.

12 Arvin AM. Humoral and cellular immunity to varicella-zoster virus: an overview. J Infect Dis 2008;197; S58-60.

13 Distler $E$, Schnürer $E$, Wagner $E$ et al. Recovery of varicella-zoster virus-specific $t$ cell immunity after $t$ cell-depleted allogeneic transplantation requires symptomatic virus reactivation. Biol Blood Marrow Transplant 2008;14;P1417-24.

14 Steain M, Sutherland JP, Rodriguez M et al. Analysis of T cell responses during active varicella-zoster virus reactivation in human ganglia. J Virol 2014:88;2704-16.
15 Wada-Shimosato Y, Tanoshima R, Hiratoko K et al. Effectiveness of acyclovir prophylaxis against varicella zoster virus disease after allogeneic hematopoietic cell transplantation: A systematic review and meta-analysis. Transpl Infect Dis 2019;21:e13061.

16 Tomblyn M, Chiller T, Einsele H et al. Guidelines for preventing infectious complications among hematopoietic cell transplantation recipients: a global perspective. Biol Blood Marrow Transplant 2009;15:1143-238.

17 Ogonek J, Juric MK, Ghimire S et al. Immune reconstitution after allogeneic hematopoietic stem cell transplantation. Front Immunol 2016;7:507.

18 Offidani M, Corvatta L, Olivieri A et al. A predictive model of varicella-zoster virus infection after autologous peripheral blood progenitor cell transplantation. Clin Infect Dis 2001;32:1414-22.

Address for correspondence: Dr Amy Edwards, Alan Naftalin Centre for Education, Newham University Hospital, Glen Road, London E13 8SL, UK.

Email: amy.edwards12@nhs.net

Twitter:@A_E_Edwards 